Meta

Journal des tradlucteurs

Translators' Journal

\title{
Étude terminologique du mot " solid-state » et problèmes de traduction connexes
}

\section{Diman Khlok}

Volume 21, numéro 4, décembre 1976

URI : https://id.erudit.org/iderudit/003312ar

DOI : https://doi.org/10.7202/003312ar

Aller au sommaire du numéro

Éditeur(s)

Les Presses de l'Université de Montréal

ISSN

0026-0452 (imprimé)

1492-1421 (numérique)

Découvrir la revue

Citer cet article

Khlok, D. (1976). Étude terminologique du mot « solid-state » et problèmes de traduction connexes. Meta, 21(4), 264-274. https://doi.org/10.7202/003312ar d'utilisation que vous pouvez consulter en ligne.

https://apropos.erudit.org/fr/usagers/politique-dutilisation/ 


\section{ÉTUDE TERMINOLOGIQUE DU MOT «SOLID-STATE» ET PROBLĖMES DE TRADUCTION CONNEXES}

Il serait superflu de vouloir souligner l'importance du rôle que jouent les composants électroniques dans tous les domaines. Depuis les années cinquante, ceux-ci ont connu une évolution très rapide, caractérisée notamment par une orientation vers la miniaturisation. Même s'ils restent très peu connus du grand public, les composants électroniques n'en sont pas moins omniprésents, voire indispensables. Ils interviennent dans la vie quotidienne à tous les niveaux, qu'il s'agisse du chauffage des immeubles, du fonctionnement des ordinateurs ou bien encore de la commande des machines-outils utilisées dans l'industrie. Le mot " solid-state " sert à qualifier, en anglais, une catégorie de composants électroniques. La publicité s'ingénie à employer ce mot, d'une façon fort désinvolte d'ailleurs, car c'est un terme passe-partout qui paraît bien savant. Cet emploi abusif pose quelques problèmes de traduction, en particulier lorsqu'il s'agit de rendre " solidstate " en français par un équivalent unique. Faut-il dire "état solide "? Ce serait une solution trop facile, comme la présente étude terminologique va nous le montrer.

\section{Étude terminologique}

En parcourant tous les dictionnaires spécialisés de physique et d'électronique cités dans la liste des ouvrages de référence, nous constatons qu'il existe deux genres de définitions : les définitions très exhaustives et explicites d'une part et les définitions incomplètes et restrictives d'autre part. Pour délimiter avec précision le champ sémantique de ce mot en anglais, nous allons examiner une définition qui soit aussi complète que possible, puis analyser les différentes acceptions. Dans toutes les sources, on introduit la notion de " solid-state " en définissant "solid-state physics ", c'est-à-dire la branche de la physique qui traite de l'état solide. "Solid-state " est employé comme adjectif, et le Webster ${ }^{1}$ en donne la définition suivante :

" solid-state : adj. 1. relating to the properties, structure or reactivity of solid materials; esp.: relating to the arrangement or behavior of ions, molecules, nucleons, electrons, and holes in the crystals of a substance (as a semiconductor) or to the effect of crystal imperfections on the properties of a solid substance (solid-state physics). 2. utilizing the electric, magnetic or

1. Voir chiffre correspondant dans la bibliographie. 
photic properties of solid materials : not utilizing electron tubes (e.g. solidstate stereo). "

La première acception présente un aspect scientifique et porte davantage sur l'explication d'un phénomène au niveau de la structure moléculaire de la matière. La seconde, par contre, s'attache plutôt à l'aspect technique, dont la publicité fait trop grand usage. L'aspect commercial qui en découle fausse la notion transmise dans la deuxième acception. The International Dictionary ${ }^{2}$, par rapport à The Encyclopedia of Physics ${ }^{3}$, donne une définition moins complète, mais très concise :

"solid-state physics: Generally speaking, the branch of physics which deals with the structure and properties of solids. The crystallography which deals with metals, alloys, ionic crystals, cohesive forces and band structures. The physiology of solids covers the specific heats, the thermal vibrations, the thermal and electrical conductivity, the intrinsic conductivity and the magnetic and dielectric properties. The pathology of solids studies the impurities in semiconductivity, the plasticity, the lattice defects, the color centers, the dislocation theory and the crystal growth."

A New Dictionary of Physics ${ }^{4}$, dans sa définition de " solid-state physics ", est à la fois concis et exhaustif. D'autres ouvrages de référence ${ }^{5,6}$ offrent toutefois des définitions qui, de par leur caractère restrictif, sont équivoques :

" solid-state physics: The branch of physics that deals with the structure and properties of solids, including semiconductors. ${ }^{2}$

Ce genre de définition est inexact, car il limite la définition de " solid-state " aux semiconducteurs. À la lumière de ces constatations, il faut donc d'abstenir de traduire " solid-state physics " par " physique des semiconducteurs". Les équivalents français pour "solid-state physics" sont plutôt " physique du solide ", " physique des solides" et " physique de l'état solide ". Les spécialistes du CNRS ${ }^{7}$ emploient " physique de l'état condensé ". Ils considèrent en effet que les molécules des solides, contrairement à celles des liquides et des gaz, sont comprimées et forment ainsi un état " condensé ". Après avoir examiné les différents équivalents de "solid-state physics ", nous allons passer en revue les divers substantifs qui peuvent être associés à l'adjectif "solid-state ", et nous essayerons d'étudier les équivalents français de chacun d'eux. Nous nous efforcerons en dernier lieu de déterminer s'il existe une seule et unique traduction pour * solid-state " ou si cette dernière varie en fonction du substantif employé.

Étude des différents substantifs associés à "solid-state" et discussion des traductions

Nous allons procéder systématiquement, à partir des éléments les plus petits pour aboutir aux appareils, en passant par les composants et les circuits. 
1. Solid-state materials. - Solid-state electronics ${ }^{8}$ est un ouvrage qui est censé traiter de l'"électronique des solides", mais il n'aborde en fait que les "semiconducteurs". "Solid-state" est donc considéré comme un générique qui couvre tous les semiconducteurs. Par "solid-state materials", l'auteur désigne l'ensemble des corps ayant des propriétés identiques à celles du germanium et du silicium, deux éléments (en chimie : corps purs simples) fondamentaux dont les propriétés ont permis d'élaborer la théorie des semiconducteurs. En français, " semiconducteur " peut être substantif ou adjectif. Ainsi " matériau semiconducteur "peut servir à désigner le germanium et le silicium. Mais ces deux éléments et leurs oxydes ne sont pas les seuls semiconducteurs, car il existe d'autres " oxydes métalliques ", notamment l'oxyde de cuivre, ainsi que des " alliages " aux propriétés semiconductrices. En ne traitant que des semiconducteurs, l'auteur restreint le sens de "solid-state ", qui devient alors un spécifique synonyme de a semiconductor ". "Solid-state " étant remplacé par "semiconductor", nous obtenons les équivalences suivantes :

semiconductor material (S)

(solid-state material) $(\mathrm{G})$

$=$ semiconducteur (subst.) $\mathrm{G}$ matériau semiconducteur (adj.) $G$

Compound semiconductor material $(\mathbf{S})=$ semiconducteur (subst.) $\mathrm{G}$ (compound solid-state material) $(\mathrm{G}) \quad$ composé semiconducteur (adj.) $\mathrm{G}$

2. Solid-state component. - La définition de ce terme ("A component whose operation depends on the control of electrical or magnetic phenomena in solids, such as transistor, crystal diode or ferrite device. ") est commune à trois ouvrages ${ }^{4,6,9}$. M. Ginguay ${ }^{10}$ donne pour équivalent français " composant semiconducteur ". Ici encore, on se limite aux semiconducteurs, et le terme français, dont la valeur est ainsi diminuée, demeure un spécifique alors que le terme anglais est un générique. On pourrait à la rigueur employer " composant électronique ", mais on se trouverait alors à trop étendre son champ sémantique (les composants électroniques englobent aussi les tubes à vide), et la traduction n'en serait que plus ambiguë. L'anglais aurait, pour sa part, utilisé, "electronic component" au lieu de " solid-state component". Ce générique n'est donc pas à conseiller.

solid-state component (G)

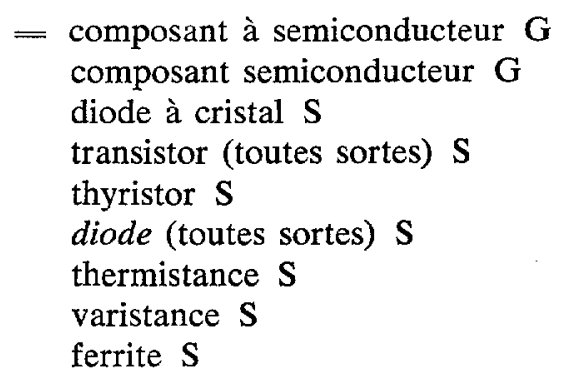

3. Solid-state device. - L'Encyclopedia Americana ${ }^{11}$ précise:

"Solid-state: A term that was first introduced in electronics to distinguish solid semiconductor devices from their vacuum-tube counterparts."

H.J. Gray et A. Isaacs ${ }^{4}$ définissent "solid-state device" de la façon suivante : 
- An electronic device consisting chiefly or exclusively of semiconducting materials or components. "La deuxième partie de cette phrase (" or exclusively... ") restreint le sens et, par conséquent, diminue la valeur de la définition. L'anglais dirait alors " semiconductor device", terme qui figure dans tous les dictionnaires cités. La définition de R.F. Graf ${ }^{6}$ est plus étendue et cadre mieux avec la définition de "solid-state physics", qui englobe les propriétés magnétiques, opto-électriques et opto-électroniques :

"Any element that can control current without moving parts, heated filaments or vacuum gaps. All semi-conductors are solid-state devices, although not all solid-state devices are semiconductors (e.g. transformers)."

Nous découvrons un nouvel aspect: on considère l'existence de parties non mobiles, par opposition aux parties mobiles. Diverses revues publiées en français, ainsi que l'Encyclopédie Quillet ${ }^{12}$, insistent sur ce même aspect pour désigner la réalité, c'est-à-dire que les pièces ne bougent pas, donc sont "statiques ". On parle alors de "dispositifs statiques", terme qui offre l'avantage d'être un générique cette fois-ci. Le Bulletin signalétique du $\mathrm{CNRS}^{7}$ parle également de " dispositifs à corps solides ". En considérant le premier aspect, soit celui de la conductivité électrique (semiconduction), on trouve dans plusieurs ouvrages ${ }^{12,13}$ les spécifiques suivants : " dispositif à semiconducteur ", " dispositif à transistor " " dispositif transistorisé ". Mais si l'on pense aux propriétés magnétiques, c'est-àdire aux ferrites, ils demeurent des spécifiques. Pour illustrer tout ceci, nous allons puiser un exemple concret dans la rubrique "solid-state relay *.

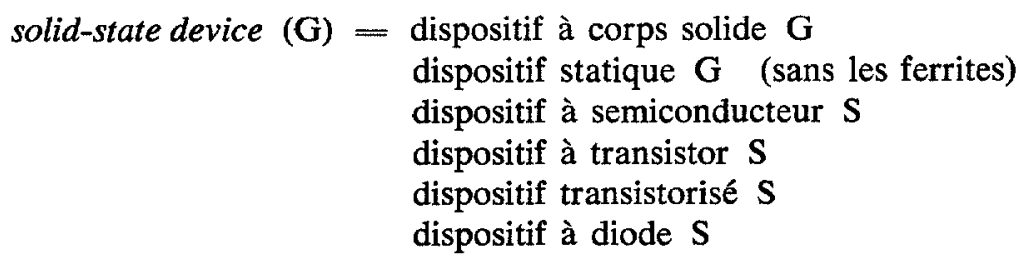

4. Solid-state relay. - H.J. Gray et A. Isaacs ${ }^{4}$ nous renvoient, à partir de ce terme, à la vedette * relay ", qu'ils définissent comme suit :

" An electrical device in which one electrical phenomenon controls the switching on or off of an independent electrical phenomenon. There are many types of relays, most of which are either electromagnetic or solid-state relays. "

Pour " solid-state relay ", on trouve: "Any true solid-state relay has all its components made from solid-state devices. "On explique le fonctionnement en mentionnant que la commutation est assurée par un thyristor interrupteur, et on reprend plus loin : "such a relay has no moving parts [...] and has advantages over electromagnetic relays... " Plutôt que de mettre l'accent sur le fonctionnement des relais en question au moyen de dispositifs à semiconducteurs, le texte intégral de cette définition nous montre que l'anglais insiste davantage sur le fait que les parties ne sont pas mobiles, c'est-à-dire statiques, caractéristique dont il nous 
présente tous les avantages. En français, si l'on cherche à " relais ", on constate que partout, notamment dans l'Encyclopédie Quillet ${ }^{12}$ et de Dictionnaire technique de la construction électrique ${ }^{14}$, il s'agit de "relais statiques", et non de "relais transistorisés ", comme on le trouve dans certaines brochures ou autres traductions techniques. En téléphonie, on parle à la rigueur de "relais électroniques " pour les différencier des relais électromécaniques.

solid-state relay $(\mathrm{G})=$ relais statique $\mathrm{G}$

équivalence parfaite

(à la rigueur) relais électronique $\mathrm{G}$

5. Solid-state circuit. - Le McGraw-Hill Dictionary of Scientific and Technical Terms" donne pour ce terme la définition suivante: "Complete circuit formed from a simple bloc of semiconductor material. " "Circuit à semiconducteur " est communément employé dans les textes français. Certains circuits, entièrement composés de semiconducteurs, sont appelés " circuits à semiconducteurs " et sont constitués par une grande variété de composants à semiconducteurs : diodes, transistors, etc. Lorsque le circuit ne comprend que des transistors, ce qui est rarement le cas, on parle de " circuits transistorisés". Lorsqu'il s'agit de circuits intégrés, l'anglais emploie explicitement a integrated circuits ". Si le circuit à semiconducteur est un circuit intégré, on parle alors plus précisément de "solid-state integrated circuit ". L'ouvrage de R.F. Graf ${ }^{6}$ donne, pour cette dernière entrée, la définition suivante: "The class of integrated components in which only solid-state materials are used. "Le terme "materials" couvre les divers semiconducteurs.

$$
\begin{gathered}
\text { solid-state circuit }(\mathrm{S})=\text { circuit à semiconducteur } \mathrm{S} \\
\text { (ou circuit transistorisé) } \mathrm{S} \\
\text { équivalence parfaite }
\end{gathered}
$$

$$
\begin{aligned}
& \text { solid-state integrated circuit }(\mathrm{S})=\text { circuit intégré à semiconducteur } \mathrm{S} \\
& \text { équivalence parfaite }
\end{aligned}
$$

\section{integrated circuit $(\mathrm{S})=$ circuit intégré $\mathrm{S}$} équivalence parfaite

6. Solid-state computer. - R.F. Graf ${ }^{6}$ définit ce terme comme suit: "A computer built primarily from solid-state electronic circuit elements. "M. Ginguay ${ }^{10}$ donne, pour ce terme, l'équivalent français " ordinateur transistorisé ", trop restrictif car les circuits de commande ne sont pas seulement constitués par des transistors. Il serait plus pertinent de parler de circuits intégrés, et il s'agirait done d'un * ordinateur à circuits intégrés ".

solid-state computer $(\mathbf{S})=$ ordinateur à circuits intégrés $\mathrm{S}$ meilleure équivalence que ordinateur transistorisé $\mathrm{S}$ 
7. Solid-state laser. - S. Marshall ${ }^{15}$ parle de " solid-state laser ", en soulignant toutefois que " the term solid-state implies lasers using cristalline or glass doped with suitable ions; it does not include semiconductor laser. "En français, on distingue les lasers à gaz, les lasers liquides et les " lasers à solides" (la revue Automatisme, $\mathrm{n}^{\circ}$ 6.7, juin/juillet 1972, Dunod). Ceux-ci ne comprennent pas les lasers à semiconducteurs 13 et 16 mais seulement les lasers à cristal. Il existe plus particulièrement un laser à rubis, qui a été le premier laser à cristal.

$$
\begin{aligned}
\text { solid-state laser }(\mathrm{S})= & \text { laser à solide } \mathrm{S} \\
& \text { laser à cristal (à rubis) } \mathrm{S}
\end{aligned}
$$

8. Solid-state maser. - Markus ${ }^{5}$ donne une définition très explicite : il s'agit d'un dispositif soit à cristal, soit à semiconducteur. Par analogie avec laser, il serait possible de désigner ce maser comme un "maser à solide "ou "maser à cristal ». Un maser est pratiquement semblable à un laser, sauf que le faisceau cohérent se produit dans le domaine des ondes micrométriques, au lieu de se produire dans le visible, l'ultraviolet ou l'infra-rouge, comme c'est le cas pour le laser. Pour le maser à cristal, il peut s'agir soit d'un maser à rubis, soit d'un maser à émeraude.

solid-state maser $(\mathrm{S})=$ maser à solide (à cristal) $\mathrm{G}$

9. Solid-state rectifier. - Pour ce terme, M. Besançon donne la définition suivante :

"A rectifier provides the physical means which achieve electric rectification, comprising all the elements which connect a complete ac circuit to a complete de circuit, without being part of either."

Ici, une discussion s'impose au sujet du néologisme de l'OLF, cahier $\mathrm{n}^{\circ} 22^{17}$. L'OLF donne " redresseur à semiconducteur, avec le néologisme " redresseur en solide ", même s'il tolère " redresseur à l'état solide ". " Redresseur en solide " est un peu restrictif du point de vue de l'emploi. Les redresseurs, en effet, comme appareils de redressement du courant, existent depuis longtemps et se présentent sous de nombreuses formes. On trouve la traduction des différents noms d'appareils dans le dictionnaire technique anglais-français de Guy Malgorn. Dans l'Encyclopédie et le Dictionnaire technique de la construction électrique 12 et 14 , on parle de "redresseurs statiques", compte tenu du fait que les redresseurs à semiconducteurs, par opposition aux redresseurs électromécaniques, n'ont aucune pièce mobile. Dans le développement technique, ils constituent la suite logique des autres redresseurs qu'ils remplacent (tubes à vide et tubes à gaz). La désignation des redresseurs déjà existants s'effectue selon un principe particulier, c'est-à-dire au moyen de la préposition "à " suivie d'un nom désignant le milieu de conduction, "vide " ou " gaz " en l'occurrence. Si l'on voulait obéir au même principe, on devrait dire tube " à solide " plutôt que * en solide ", comme le suggère l'OLF. P. Sizaire ${ }^{14}$ 
conseille les appellations " redresseur au germanium " et "redresseur au silicium " pour préciser la nature du semiconducteur. Si l'on veut désigner le redresseur à semiconducteur selon son mode de fonctionnement, on l'appelle alors " redresseur à couche d'arrêt ". Cette désignation est préférable à "redresseur statique ", car ce dernier inclut aussi les "redresseurs thermo-électroniques", également statiques (aucune pièce mobile) mais qui n'ont rien à voir avec les semiconducteurs. Les "redresseurs statiques à semiconducteurs" sont aussi des "redresseurs secs".

$\begin{aligned} \text { solid-state rectifier }(\mathrm{G}) \quad & \text { redresseur statique } \mathrm{G} \\ & \text { redresseur sec } \mathrm{G} \\ & \text { redresseur à semiconducteur } \mathrm{G} \\ & \text { redresseur au silicium } \mathrm{S} \\ & \text { redresseur au germanium } \mathrm{S} \\ & \text { redresseur à couche d'arrêt } \mathrm{G} \\ & \text { redresseur statique à semiconducteur S } \\ & \text { redresseur statique à thyristor S } \\ & \text { redresseur statique à transistor } \mathrm{S}\end{aligned}$

10. Solid-state static alternator. - La définition que donne Markus ${ }^{5}$ montre bien qu'il s'agit d'un " alternateur à redresseur au silicium ".

11. Solid-state battery. - Dans des publications scientifiques, les spécialistes emploient "pile à électrolyte solide ". Il ne faut donc pas confondre "solid-state battery " avec " semiconductor battery ", qui sont des "convertisseurs photovoltaïques " (générique) parmi lesquels on compte les "photopiles" (spécifique).

\section{Discussion}

Dans sa publication, F. de Closets ${ }^{18}$ mentionne à tort "télévision solide" pour rendre ce que la publicité anglaise désigne par " solid-state TV", mettant de ce fait l'accent sur le caractère fictif de la rigidité. Le dictionnaire d'électronique des Editions Chiron ${ }^{19}$ se place d'un autre point de vue quand il traduit " solid state device " par " dispositif compact ". Quant à "solid-state physics", les dictionnaires d'électrotechnique et d'électronique ${ }^{19}$ et 20 donnent les traductions " physique du solide " et " physique de l'état solide ". G. Proul ${ }^{21}$ parle de "matériel électronique "(constitué de corps solides) pour rendre "solid-state electronics", traduction fort ambiguë d'ailleurs. Dans la référence 12 du Vocabulaire électrotechnique $e^{22}$, on trouve "transistorisé "pour " récepteur à transistors". On pourrait alors dire "poste de radio transistorisé " et " téléviseur transistorisé " pour " solidstate radio " et " solid-state TV ". L'appareil " transistorisé " ne compớte ni lampe, ni tubes. Lorsqu'on veut préciser que les châssis des appareils sont transistorisés, il importe de dire "appareils à châssis transistorisés ". En parlant d'" appareils à circuits intégrés ", on insiste sur la miniaturisation des appareils " solid-state ". 

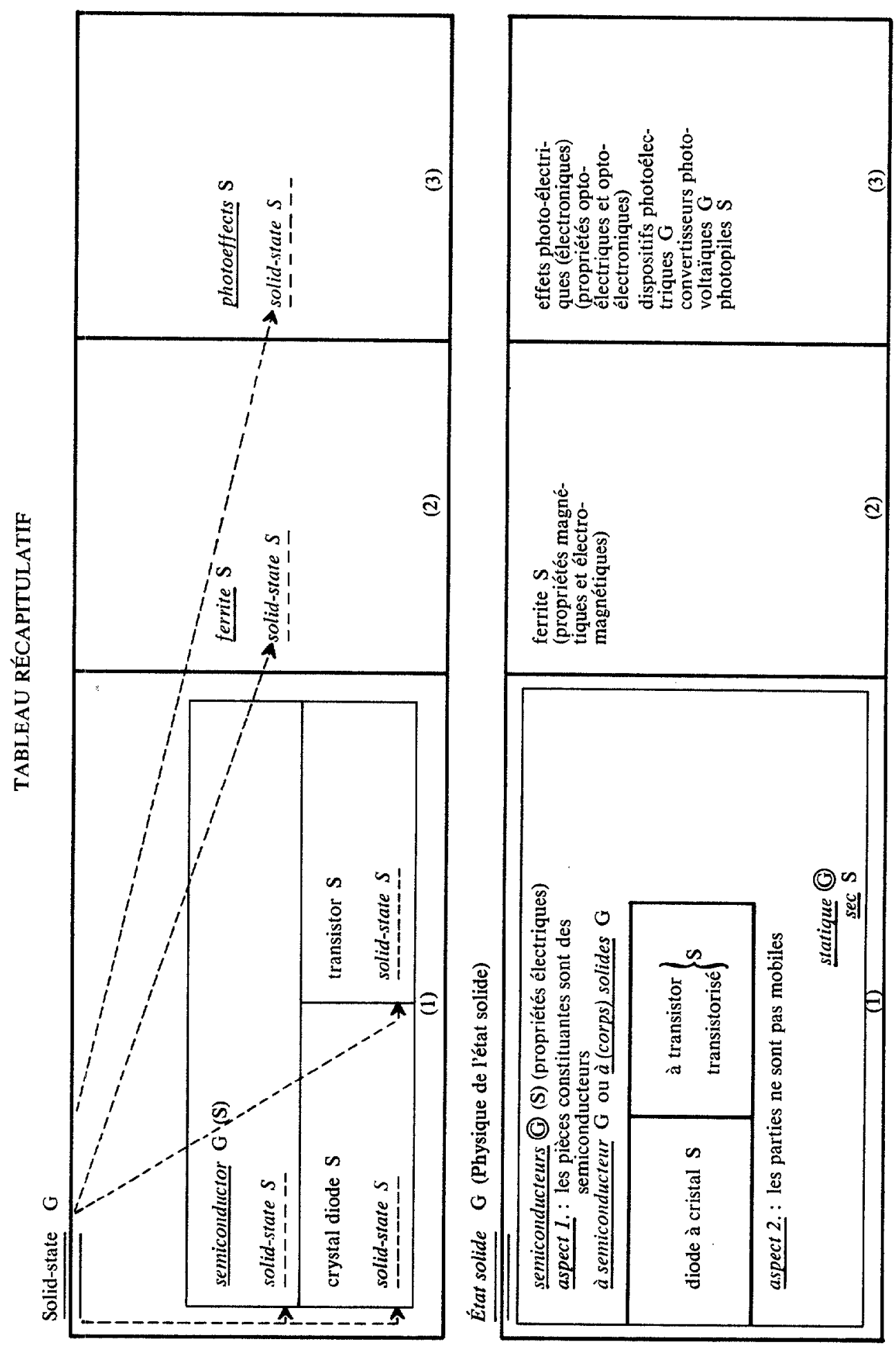
N.B. : En français, ce passage du générique au spécifique n'existe pas pour a état solide ", dont le champ sémantique est identique à celui de " solid-state " seulement dans le cas de "solid-state physics". Pour la traduction d'autres termes, on doit faire appel à des spécifiques, donc savoir exactement ce dont il s'agit dans chacun des cas. On ne dispose pas du même degré de liberté offert par " solid-state ", qui est en fait un passe-partout bien commode, surtout pour la publicité.

\section{TABLEAU DE SYNTHÈSE}

DIMAN KHLOK

1. Solid-state physics

2. Solid-state material

Compound-state material

3. Solid-state component

4. Solid-state device

5. Solid-state relay

6. Solid-state circuit

Solid-state integrated circuit Integrated circuit

7. Solid-state computer

8. Solid-state laser

9. Solid-state maser

10. Solid-state rectifier
$=$ physique du solide $\mathrm{G}$ physique des solides $G$ physique de l'état solide $G$ physique de l'état condensé $G$

$=$ semiconducteur $\mathrm{G}$ matériau semiconducteur $G$

$=$ semiconducteur $\mathrm{G}$ composé semiconducteur $\mathrm{G}$

= composant électronique $\mathrm{G}$ (Attention!) composant à semiconducteur $\mathrm{G}$ composant semiconducteur $\mathrm{G}$ diode à cristal $S$

transistor $\mathrm{S}$ thyristor $S$ diode (+ spécification) $\mathrm{S}$ thermistance $\mathrm{S}$ varistance $\mathbf{S}$ ferrite $\mathbf{S}$

$=$ dispositif à corps solide $\mathrm{G}$ dispositif à semiconducteur $S$ dispositif à transistors $\mathbf{S}$ dispositif transistorisé $\mathbf{S}$ dispositif à diode $\mathbf{S}$ dispositif statique $G$ Aspect 1

relais statique $\mathrm{G}$ (relais électronique - téléphonie) $\mathrm{G}$

$=$ circuit à semiconducteur $\mathrm{S}$ circuit transistorisé $\mathbf{S}$

$=$ circuit intégré à semiconducteur $\mathrm{S}$

$=$ circuit intégré $\mathrm{S}$

$=$ ordinateur à circuits intégrés $\mathrm{S}$ (ordinateur transistorisé) $\mathrm{S}$

$=$ laser à solide $\mathrm{S}$ laser à cristal $S$

= maser à solide $\mathrm{G}$

$=$ Aspect 1.: semiconducteurs redresseur à semiconducteur $\mathrm{G}$ redresseur au silicium $\mathbf{S}$ redresseur au germanium $S$

Aspect 2.: parties non mobiles redresseur sec $S$ ( $G$ par rapport à ceux qui suivent) redresseur statique $G$ redresseur à couche d'arrêt $\mathrm{G}$ redresseur statique à semiconducteurs redresseur statique à thyristor $\mathrm{S}$ redresseur statique à transistor $\mathrm{S}$ 
11. Solid-state alternator

12. Solid-state battery

Semiconductor battery

\section{PUBLICITÉ}

1. Solid-state radio

2. Solid-state TV
= alternateur à redresseur au silicium $\mathrm{S}$

= pile à électrolyte solide $\mathrm{S}$

= convertisseur photovoltaïque $\mathrm{G}$ photopile S

$=$ récepteur à transistors $\mathrm{G}$ poste de radio transistorisé $G$

$=$ téléviseur à circuits intégrés $\mathrm{S}$

\section{BIBLIOGRAPHIE}

1. Webster's New Collegiate Dictionary, Merriam-Webster, Toronto (Ontario) - 1974.

2. The International Dictionary of Physics and Electronics, Van Nostrand Company Inc., Toronto - 1961

3. Besançon, R.M., The Encyclopedia of Physics, Second Edition, Van Nostrand and Reinhold Company, New York - 1974.

4. Gray, H.J., and Isaacs, A., A New Dictionary of Physics, Longman, London - 1975.

5. Markus, Electronics and Nucleonics Dictionary, McGraw-Hill - 1966.

6. Graf, R.F., Modern Dictionary of Electronics, The Bobbs-Merrill Co. Inc. - 1972.

7. Bulletin signalétique du CNRS, Electricité et Electronique, vol. xxxı, nos 11 et 12, Centre de documentation, Paris - 1971.

8. Hibberd, R.G., Solid-State Electronics, collect. "Texas Instruments Electronics Series", Texas Instruments Inc., McGraw-Hill - 1968.

9. McGraw-Hill Dictionary of Scientific and Technical Terms, Daniel N. Lapedes, Editor in Chief, McGraw-Hill - 1974.

10. Ginguay, M., Dictionnaire d'informatique - anglais/français, Masson, Paris - 1970.

11. Encyclopedia Americana, Americana Corporation, New York - 1973 (entrée "Electronic").

12. Encyclopédie des sciences industrielles, tome 1: "Electricité et Electronique - Généralités" tome 2: «Electricité et Electronique - Applications» Librairie Aristide Quillet, Paris - 1973.

13. Les Techniques de l'Ingénieur - Electronique (E) 21, rue Cassette, 75 006-Paris.

14. Sizaire, P., Dictionnaire technique de la construction électrique, Eyrolles, Paris -1968 .

15. Marshall, S., Laser Technology and Applications, McGraw-Hill - 1968.

16. Les Semiconducteurs, collection "Dictionnaire international de Science et de Terminologie ", DST-1, Franco Angeli Editore, Milano - Entreprise Moderne d'Edition, Paris - 1971.

17. Lexique anglais-français de l'électronique au Québec, Office de la langue française, Cahiers de l'OLF, $\mathrm{n}^{\circ} 22$, Gouvernement du Québec.

18. de Closets, F., Une nouvelle race de composants: les $C C D$, (composants à charge de couple), INFORMATECH, p. 1184, photocopies.

19. Dictionnaire anglais-français d'électronique avec définition des termes, Editions Chiron, Paris - 1967.

20. Piraux, $\mathrm{H}$., Dictionnaire anglais-français de l'électrotechnique et de l'électronique, Eyrolles, Paris - 1972.

21. Proulx, G., Dictionnaire anglais-français de l'électronique et des télécommunications, Bélisle, Québec - 1969. 
22. Bureau de normalisation du Québec, Norme Vocabulaire électrotechnique, groupe 63, nos 63,20 et 115 .

23. QUE SAIS-JE ?, L'électronique, PUF, Paris - 1973.

24. Sarbacher, R.I., Encyclopedic Dictionary of Electronics and Nuclear Engineering, Prentice-Hall Inc., Englewood Cliffs, N.J. - 1959.

25. Funk and Wagnalls Dictionary of Electronics, Funk and Wagnalls, New York - 1969.

26. Randle, G.R., Electronic Industries - A guide to information sources, Management Information Guide - 1968.

27. McGraw-Hill Encyclopedia of Science and Technology, vol. 12, McGraw-Hill - 1971.

28. Encyclopédie internationale des Sciences et des Techniques, Presses de la Cité, Paris - 1973 Larousse, distributeur exclusif pour l'étranger.

29. La Science contemporaine, tome 2: "Les sciences physiques et leurs applications ", Larousse, Paris - 1965

30. Péricone, L., L'électronique à votre service, Publications Perlor-Radio, Paris - 1971.

31. Weick, C.B., Principles of Electronic Technology, McGraw-Hill - 1969.

32. a) Grabowski, B., Les fonctions de l'électronique, collect. "GEFOP ", tome 1: "Diodes et dipôles ", Dunod, Paris - 1974

32. b) Grabowski, B., Les fonctions de l'électronique, collect. "GEFOP ", tome 2: "Tripôles actifs ", Dunod, Paris - 1974.

33. Lenk, J. D., Handbook of Practical Solid-State Trouble Shooting, Prentice-Hall Inc., Englewood Cliffs, N.J. - 1971.

34. Fritzche, H., Les semi-conducteurs amorphes, INFORMATECH, photocopies.

35. King, G.G., Dictionnaire français-anglais d'électronique et de physique nucléaire, Dunod, Paris - 1961

36. American Standard (American Institute of Electrical Engineers), Definitions of Electrical Terms, Group 70: "Electron devices " - 1957.

37. Commission Electrotechnique Internationale, Vocabulaire électrotechnique international, Groupe 07: «Electronique ».

38. Langeyl, B., Introduction à la physique du laser, Eyrolles, Paris - 1968.

39. Revues spécialisées de physique et d'électronique. 